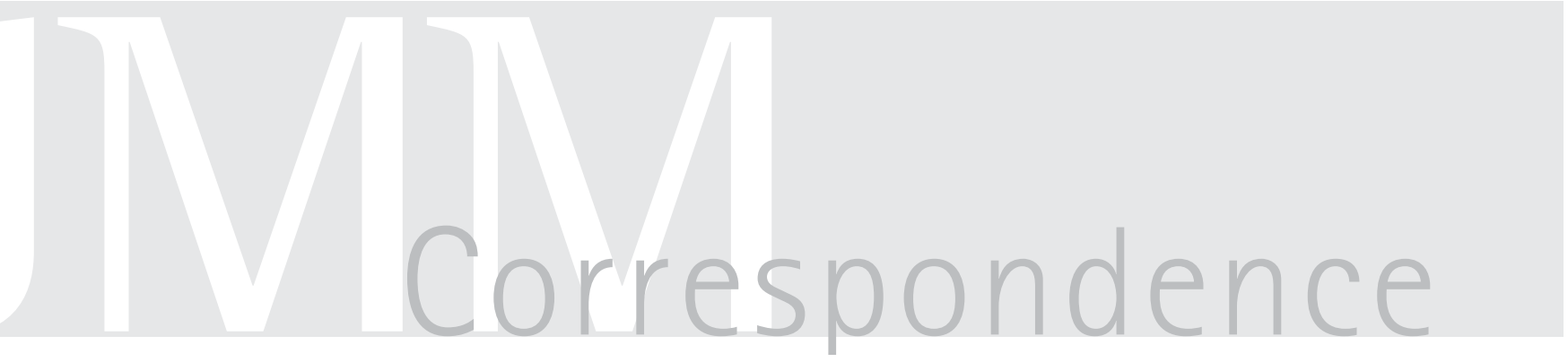

\title{
Clinical insignificance of $(1 \rightarrow 3)$ - $\beta$-D-glucan in early diagnosis of invasive pulmonary aspergillosis in a patient with chronic obstructive pulmonary disease
}

Invasive pulmonary aspergillosis is a serious threat that primarily affects immunocompromised patients, those who are severely neutropenic under cancer chemotherapy or bone-marrow transplantation, or those with acquired immunodeficiency syndrome (AIDS) (Kristan et al., 2002; Aliff et al., 2003). Also, immunocompetent patients with chronic obstructive pulmonary disease (COPD), especially those under corticosteroid therapy, have recently been implicated in the appearance of invasive aspergillosis. However, early diagnosis of invasive pulmonary aspergillosis is very difficult (Kristan et al., 2002).

Here we describe a patient with COPD who developed rapidly progressive respiratory failure and died after short-term corticosteroid treatment, in whom poor laboratory findings overwhelmed the clinical suspicion of invasive pulmonary invasive aspergillosis, which was confirmed only at post-mortem.

An 83-year-old man with a long history of COPD was admitted because of worsening dyspnoea and productive cough. He had been treated with an anticholinergic inhalation of oxitropium bromide and had repeated respiratory exacerbations resulting from bronchitis, which had improved every time with both short-term intravenous corticosteroid and antimicrobial therapy. On admission, severe wheezes were heard bilaterally. A chest radiograph was unremarkable, with pleural thickening as a sequela to tuberculosis of youth and emphysematous change. Arterial blood gases whilst breathing room air showed $\mathrm{pH}$ 7.41; $\mathrm{pCO}_{2}, 33 \mathrm{mmHg} ; \mathrm{pO}_{2}, 44 \mathrm{mmHg}$; and $\mathrm{HCO}_{3}^{-}, 17 \mathrm{mmol} \mathrm{l}^{-1}$. His leukocyte count was $6500 \mu \mathrm{l}^{-1}$ and C-reactive protein was $3 \cdot 2 \mathrm{mg} \mathrm{dl}^{-1}$. Left nephrectomy had been done in his youth and the remaining kidney had a 20-year history of diabetic nephropathy. His blood urea nitrogen was
$38.1 \mathrm{mg} \mathrm{dl}^{-1}$, serum creatinine was $1.6 \mathrm{mg}$ $\mathrm{dl}^{-1}$ and creatinine clearance was $20 \mathrm{ml}$ $\mathrm{min}^{-1}$; these values reflect his history.

Therapy with methylprednisolone $(80 \mathrm{mg}$ day $\left.^{-1}\right)$ and cefmetazole $\left(2 \mathrm{~g} \mathrm{day}^{-1}\right)$ was initiated, but his bronchospasm and dyspnoea continued. On day 7 , he experienced an acute dyspnoea attack with blood-tinged sputum. A bolus of hydrocortisone $(300 \mathrm{mg}$ ) was administered and methylprednisolone was increased to $240 \mathrm{mg}$ per culture. Repeated sputum culture yielded only methicillin-resistant Staphylococcus aureus (MRSA), which was not likely to be the pathogen responsible. Blood-stained sputum raised the suspicion of aspergillosis, but serological evidence was poor $\left[(1 \rightarrow 3)-\beta\right.$-D-glucan at $15 \mathrm{pg} \mathrm{ml}^{-1}$ and the latex agglutination test for galactomannan was negative]. As empiric itraconazole seemed to be ineffective during treatment with an $\mathrm{H}_{2}$-receptor blocker, fluconazole was initiated instead (Fig. 1). Intensification of therapy did not improve his respiratory failure. Methylprednisolone was decreased gradually, as the increase did not seem to improve the patient's condition.

On day 15 , $(1 \rightarrow 3)-\beta$-D-glucan was 292 pg $\mathrm{ml}^{-1}$ (Fig. 1). A chest radiograph showed diffuse alveolar infiltrates at bilateral base. With a strong clinical suspicion of invasive pulmonary aspergillosis, although the latex agglutination test for galactomannan remained negative, amphotericin $\mathrm{B}$ was loaded immediately and increased rapidly to $1 \mathrm{mg}$ ( $\mathrm{kg}$ body weight $)^{-1}$ day $^{-1}$, despite the patient's impaired renal function. On day 19 , because of respiratory exacerbation with haemoptysis, he was intubated and ventilated with positive airway pressure, but gas exchange deteriorated. He died on the twenty-first hospital day.

An autopsy confirmed the presence of severe pulmonary emphysema in both lungs and showed old organized tuberculosis in the left upper lobe. The diagnosis of invasive pulmonary aspergillosis was confirmed, with identification of numerous branchedseptal hyphae in extensive areas of necrotizing bronchopneumonia, spreading throughout whole-lung regions. Culture of the pulmonary specimen yielded Aspergillus fumigatus and multi-drug-resistant Burkholderia cepacia. Aspergillus hyphae were also seen in the thyroid, suggesting haematological dissemination. Shortly before death, serum analysis revealed $(1 \rightarrow 3)-\beta$-D-glucan at $1879 \mathrm{pg} \mathrm{ml}^{-1}$ and the latex agglutination test for galactomannan became positive. Retrospective analysis suggested the onset of invasive aspergillosis on day 7 , when respiratory deterioration and blood-stained sputum developed.

COPD, especially under steroid treatment, has recently been reported to have a rare but significant association with invasive pulmonary aspergillosis (Conesa et al., 1995; Pittet et al., 1996). As trials with regular use of inhaled steroids in COPD are under way in European countries, and because short-term use of intravenous corticosteroids is effective for acute exacerbation of COPD, invasive aspergillosis should be considered carefully as a differential diagnosis of pulmonary infection in COPD patients undergoing corticosteroid treatment (Conesa et al., 1995; Pittet et al., 1996). However, difficulty in diagnosis, along with the nephrotoxicity of amphotericin B (the only effective intravenous drug that is commercially available), makes it almost impossible to save patients in the fulminant course of the disease.

Histopathological or mycological diagnosis by transbronchial biopsy or bronchoalveolar lavage under bronchoscopy is confirmative, but is often hazardous for patients with immunosuppression or severe respiratory distress (Kristan et al., 2002). Isolation of Aspergillus or detection of galactomannan from respiratory secretions may represent colonization, rather than disease. Therefore, diagnosis is usually based on a combination 


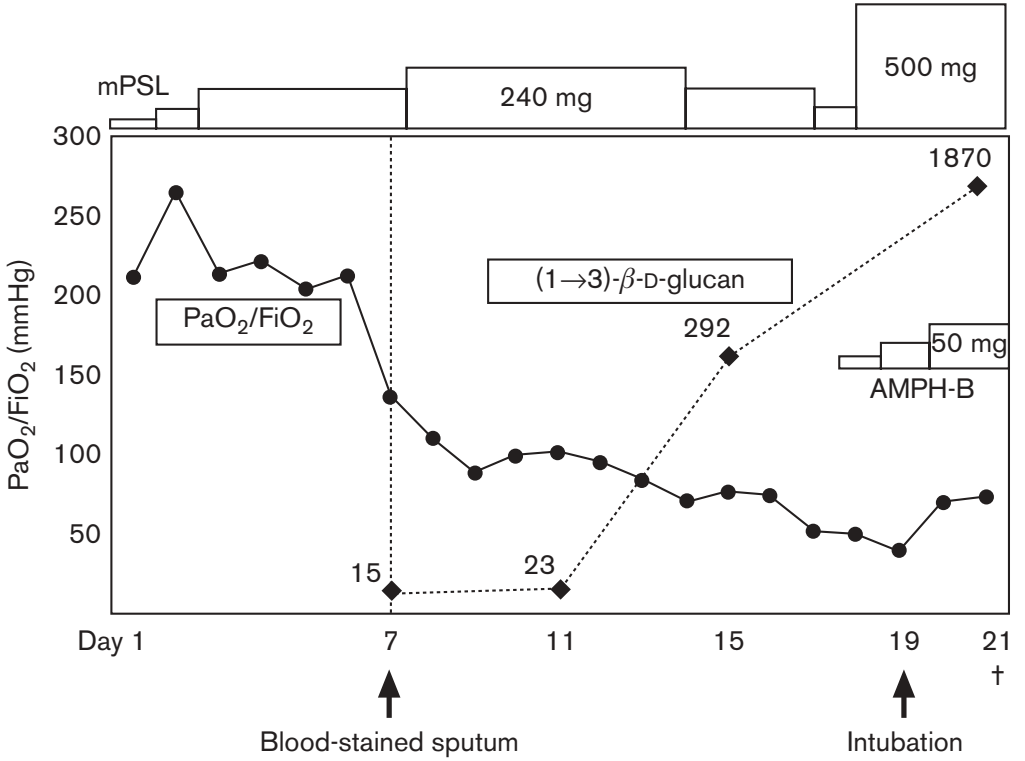

Fig. 1. Clinical course and serological markers for pulmonary aspergillosis in this case. of clinical, radiological and serological findings. Regarding serodiagnostic methods, an Aspergillus-specific PCR and an ELISA for galactomannan have been reported to yield a high sensitivity, but are not yet widely available (Becker et al., 2000; Meletiadis et al., 2003). Therefore, other easy and sensitive serological tests are necessary to diagnose pulmonary aspergillosis in immunocompromised patients.

Determination of plasma $(1 \rightarrow 3)-\beta$-Dglucan with factor $\mathrm{G}$ has been reported to be a highly sensitive and specific test for invasive deep mycosis, including aspergillosis, which would benefit immunocompromised patients substantially (Obayashi et al., 1995). Measurement of serum $(1 \rightarrow 3)-\beta$-D-glucan, which is a cell-wall constituent of fungi, is used widely to diagnose deep mycosis in Japan (Teramoto et al., 2000). This method was used for this case; unfortunately, it was not possible to delay therapy for serological diagnosis of aspergillosis or the high plasma level of $(1 \rightarrow 3)-\beta$-D-glucan on day 15 . The latex agglutination test for galactomannan was used for diagnosis of pulmonary aspergillosis, but this test was finally positive only just before death. Our experience was that the measurement of plasma $(1 \rightarrow 3)-\beta$ $\mathrm{D}$-glucan and the latex agglutination test for galactomannan are both effective in diagnosis of pulmonary aspergillosis, but positivity was too late to achieve a cure. In patients with COPD, the complication of invasive pulmonary aspergillosis is rare but fatal. Thus, early diagnosis is needed to cure respiratory distress due to invasive aspergillosis. Although measurement of serum $(1 \rightarrow 3)$ - $\beta$-D-glucan may be better than previous serological methods for diagnosis and therapeutic monitoring of pulmonary aspergillosis, the test is not yet significant in the early diagnosis of pulmonary aspergillosis.

Seiko Okada, ${ }^{1}$ Shinji Teramoto, ${ }^{2}$ Hajime Takizawa, ${ }^{1}$ Yasuyoshi Ouchi ${ }^{2}$ and Rokuo Matsuoka ${ }^{3}$

Departments of Respiratory Medicine ${ }^{1}$ and Geriatric Medicine ${ }^{2}$, University of Tokyo Hospital, 7-3-1 Hongo Bunkyo-ku Tokyo, 113-8655, Japan

${ }^{3}$ Department of Respiratory Medicine, Showa General Hospital, Tokyo, Japan

Correspondence: Shinji Teramoto (shinjit-tky@umin.ac.jp)

Aliff, T. B., Maslak, P. G., Jurcic, J. G., Heaney, M. L., Cathcart, K. N., Sepkowitz, K. A. \& Weiss, M. A. (2003). Refractory Aspergillus pneumonia in patients with acute leukemia: successful therapy with combination caspofungin and liposomal amphotericin. Cancer 97, 1025-1032.

Becker, M. J., de Marie, S., Willemse, D., Verbrugh, H. A. \& Bakker-Woudenberg, I. A. J. M. (2000). Quantitative galactomannan detection is superior to PCR in diagnosing and monitoring invasive pulmonary aspergillosis in an experimental rat model. J Clin Microbiol 38, 1434-1438.

Conesa, D., Rello, J., Valles, J., Mariscal, D. \& Ferreres, J. C. (1995). Invasive aspergillosis: a life-threatening complication of short-term steroid treatment. Ann Pharmacother 29, $1235-1237$.

Kristan, S. S., Kern, I. \& Mušič, E. (2002). Invasive pulmonary aspergillosis. Respiration 69, 521-525.

Meletiadis, J., Melchers, W. J., Meis, J. F., Van Den Hurk, P., Jannes, G. \& Verweij, P. E. (2003). Evaluation of a polymerase chain reaction reverse hybridization line probe assay for the detection and identification of medically important fungi in bronchoalveolar lavage fluids. Med Mycol 41, 65-74.

Obayashi, T., Yoshida, M., Mori, T. \& 10 other authors (1995). Plasma ( $1 \rightarrow 3)$ - $\beta$-D-glucan measurement in diagnosis of invasive deep mycosis and fungal febrile episodes. Lancet 345, 17-20.

Pittet, D., Huguenin, T., Dharan, S., SztajzelBoissard, J., Ducel, G., Thorens, J. B., Auckenthaler, R. \& Chevrolet, J. C. (1996). Unusual cause of lethal pulmonary aspergillosis in patients with chronic obstructive pulmonary disease. Am J Respir Crit Care Med 154, 541-544.

Teramoto, S., Sawaki, D., Okada, S. \& Ouchi, Y. (2000). Markedly increased plasma $(1 \rightarrow 3)$ $\beta$-D-glucan is a diagnostic and therapeutic indicator of Pneumocystis carinii pneumonia in a non-AIDS patient. J Med Microbiol 49, 393-394. 